\title{
Les biens non substituables dans l'occident médiéval
}

Non substitutable goods in the Medieval West

\section{Gil Bartholeyns}

\section{OpenEdition}

1 Journals

\section{Édition électronique}

URL : https://journals.openedition.org/tc/6298

DOI : $10.4000 /$ tc. 6298

ISBN : 1952-420X

ISSN : 1952-420X

\section{Éditeur}

Éditions de l'EHESS

\section{Édition imprimée}

Date de publication : 15 juin 2012

Pagination : 104-121

ISBN : 2-7351-1512-7

ISSN : 0248-6016

\section{Référence électronique}

Gil Bartholeyns, «Les biens non substituables dans l'occident médiéval », Techniques \& Culture [En ligne], 58 | 2012, mis en ligne le 17 décembre 2012, consulté le 29 septembre 2022. URL : http:// journals.openedition.org/tc/6298; DOI : https://doi.org/10.4000/tc.6298 


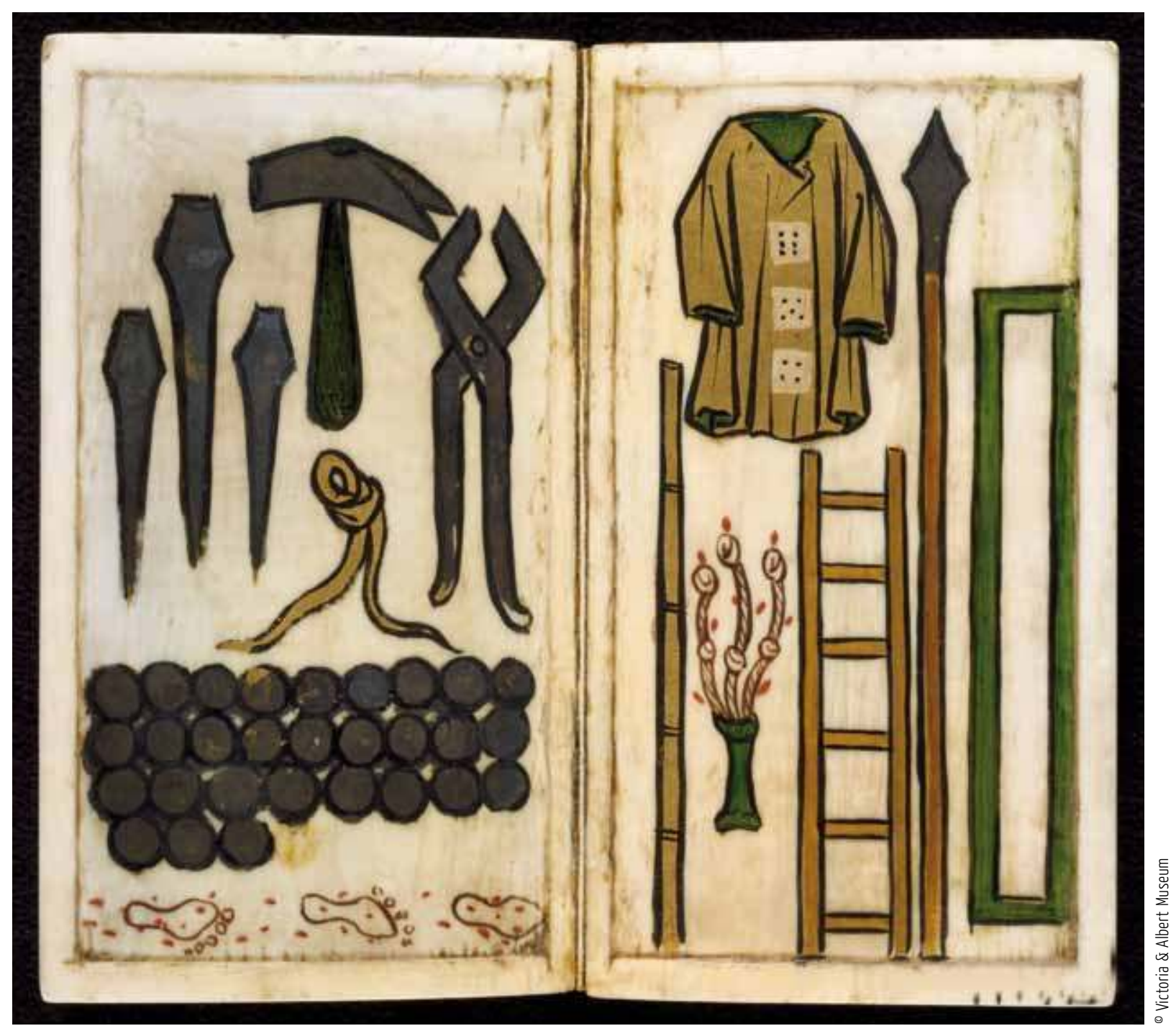




\section{LES BIENS NON SUBSTITUABLES DANS L'OCCIDENT MÉDIÉVAL}

Les céréales étaient indispensables aux hommes de l'Europe médiévale qui ne connaissaient pas le maïs ou la pomme de terre. Le fer et la pierre l'étaient à leur manière pour la société féodale. Comme dans toutes les sociétés, on ne pouvait se passer de certains biens, soit pour la vie même, soit pour la reproduction de la société ou l'expression de celle-ci' ${ }^{1}$.

Le culte chrétien, central dans le monde médiéval, ainsi que les grandes institutions telles que la royauté, donnaient de nombreuses occasions de créer et d'expérimenter le caractère indispensable de certains biens: des objets étaient exclusivement dédiés à des rituels très précis; les reliques étaient en théorie uniques, etc. La production économique engendrait également des objets volontairement singularisés. Les réalisations des artisans ne se valaient pas entre elles, ni les matériaux qui possèdent différentes espèces ou qualités, comme le bois ou la fourrure; mais à la fin du Moyen Âge, une nouveauté apparaît. Certains biens, souvent fabriqués massivement, comme le textile, se chargent publiquement d'identités particulières qui les rendent uniques en leur genre et par là même non substituables entre eux.

Mais en quels termes, tout d'abord, les médiévaux pensaient-ils le domaine de l'irremplaçable, puisque les mots « remplaçable » et « irremplaçable » n'apparaissent qu'au $x_{X}{ }^{e}$ siècle, en français comme dans les autres langues européennes? 


\section{Nécessaire, substituable, original}

Si l'on remonte les siècles, on s'aperçoit que le verbe « remplacer » n'est attesté que dans le courant du $\mathrm{xvI}^{\mathrm{e}}$ siècle, dans le sens de placer une chose ou une personne à la place d'une autre, ou de faire jouer à quelque chose ou à quelqu'un le rôle d'un autre. De la même manière, le mot « remplacement » n'apparaît pas avant les années 1530, et l'adjectif « inaliénable » est associé à un droit et non à un bien que l'on ne peut remettre en circulation et qui en vaudrait un autre. Quant au mot « indispensable », il ne prend son sens actuel qu'au xvII siècle, signifiant d'abord «ce dont on ne peut être dispensé », en empruntant au verbe latin dispensare qui signifiait aussi « distribuer $»^{2}$. Quels étaient donc les équivalents d'« irremplaçable », les voisinages sémantiques plus anciens?

Synonyme d'inévitable et d'indispensable, le terme necessarius (nécessaire) remplissait parfaitement ce rôle. Présent dans tous les domaines, tant matériels qu'idéels (logique, morale ou vie pratique), il était substantivé au neutre pluriel - les necessaria - soit « l'ensemble des choses nécessaires [indispensables] ». Le passage de cet adjectif dans les langues vernaculaires se fait dès le XII ${ }^{e}$ siècle en conservant le sens latin classique d'inéluctable, qualifiant la chose « dont on ne peut se passer ». Quels biens relevaient donc de la necessitas, de la «nécessité », un mot attesté en français vers 1150? Dans les textes bibliques, dont chacun était plus ou moins familier par la lecture ou l'écoute, les choses matérielles jugées les plus « nécessaires » sont la nourriture, le vêtement et le toit. Les Évangiles et les écrits de Paul sont traversés par le principe selon lequel il ne faut se soucier que du nécessaire, car tout le reste est en trop (Matthieu 6, 31-34; Marc 12, 43-44). Le couple utile/superflu des sagesses gréco-romaines se durcit avec le passage au christianisme en une opposition nécessaire/superflu, voire indispensable/interdit. C'est avant tout dans le message évangélique que le clerc ou le laïc rencontrait l'idée d'un bien, d'un acte ou d'une qualité irremplaçable.

Enfin, il semble que la notion de substitution soit essentielle. Si l'irremplaçabilité a été pensée sur le mode du minimum vital et du nécessaire dans le registre moral, elle a pu l'être également à partir du substantif bas latin substitutio, puis « substitution » (au xiII ${ }^{e}$ siècle), qui traduisent l'idée de mettre une chose à la place d'une autre (l'équivalent de « remplacement » qui apparaît trois siècles plus tard). Il n'y a pas d'antonyme ni d'adjectif indiquant l'impossibilité de remplacer, d'échanger, de permuter, d'intervertir, mais le verbe « substituer » (substituere) et le terme « substitution » pouvaient être employés dans des formulations négatives. Et le préfixe sub-indique implicitement l'infériorité du substitut par rapport au substitué (mettre sous, mettre après), du moins la non-équivalence. Or la « représentation » est un procédé essentiel dans la société médiévale. Le pape est le représentant de Dieu sur terre, son substitutus. Il se fait représenter par les légats pontificaux (surtout après la réforme grégorienne, Weiss 1995). Les ambassades et émissaires représentent à distance l'autorité des princes dans les négociations (Queller 1967). On pouvait envoyer un fidèle se marier à sa place (Gaudemet 1987 : 179-180), comme Othon de Grandson qui représente par procuration le comte de Savoie Amédée V devant Marie de Brabant en 1297 (Kusman 2005 : 124-128). Un mannequin ou un linceul vide, signalant la présence par l'absence, est exhibé sur le catafalque lors des funérailles des rois (Ginzburg 2001), et un condamné par contumace (Obarrio Moreno 2009) peut faire l'objet d'une exécution en effigie. L'une des exécutions en effigie les plus retentissantes est celle de Sigismond Malatesta en 1462 sur les marches de Saint-Pierre où un « gigantesque 


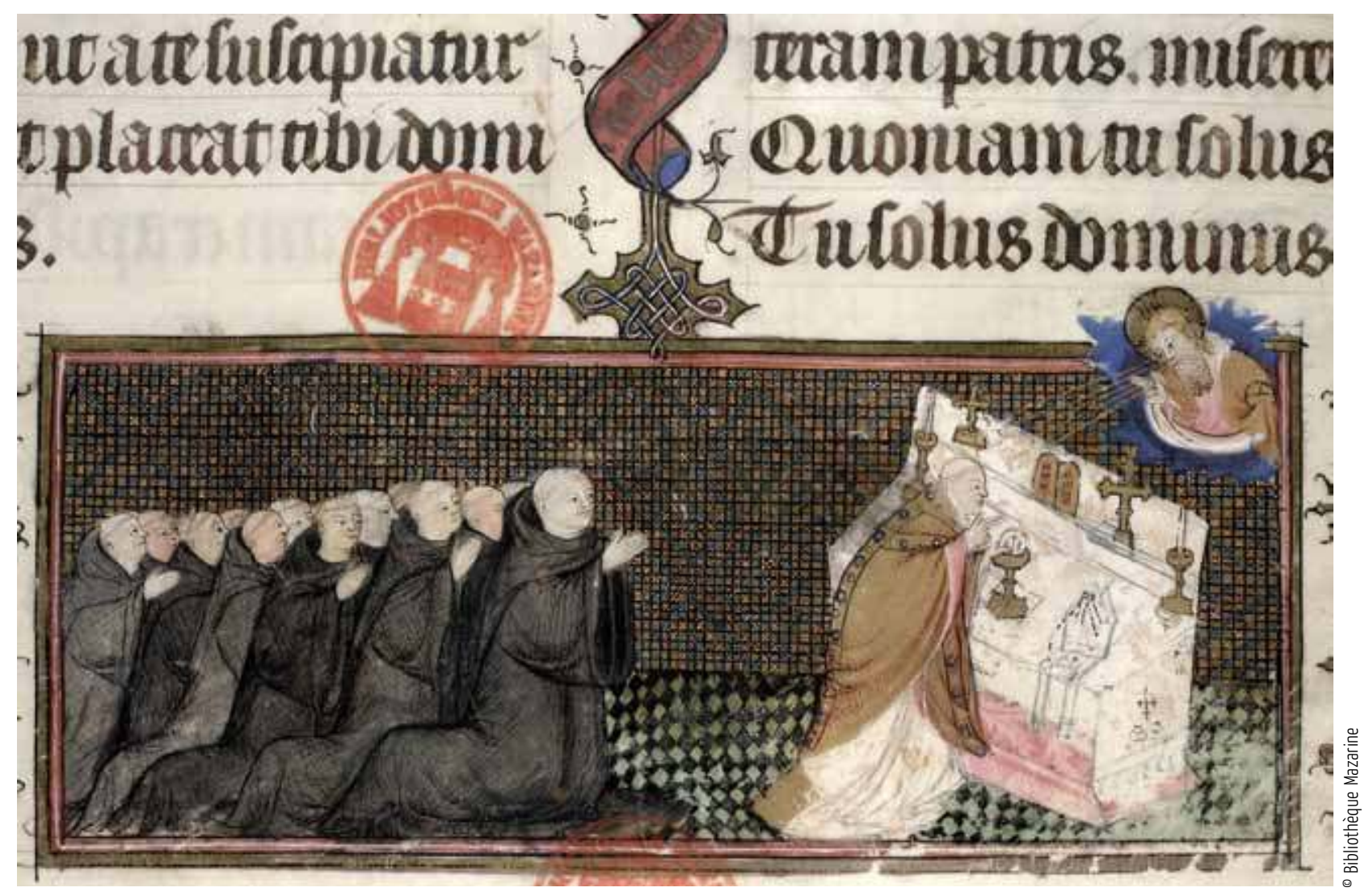

bûcher de bois sec [avait été] élevé ». Le pape Pie II raconte que l'« imago de Sigismond restitu[ait] si fidèlement les traits de l'homme et son vêtement qu'il semblait que ce fût lui en personne plutôt qu'en image $»^{3}$. Dans le cadre cultuel enfin, passé le $\mathrm{X}^{\mathrm{e}}$ siècle, le Christ, la Vierge et les saints sont de plus en plus représentés. Ils accomplissent des prodiges à travers leurs images, et certaines copies de ces images, en bois ou dessinées, possèdent elles aussi un pouvoir miraculeux (Sansterre 2011). Mais surtout, au cours de l'eucharistie, le pain et le vin sont réellement et non pas symboliquement transformés en corps et en sang du Christ, par les paroles et les gestes rituels du prêtre, en vertu de la doctrine de la transsubstantiation, antérieure au concile de Latran de 1215 mais formulée à cette occasion (Figure 1). Portée à son comble dans le cas de la consécration de l'hostie, la représentation, omniprésente dans le christianisme et dans le système féodal, permettait à un être ou à un objet (ou à une « représentation», au sens moderne qui émerge au Xive siècle) de valoir pour un autre, d'agir ou de pâtir à sa place.

Cette grande souplesse était contrebalancée par d'autres aspects fondateurs des institutions médiévales.

Certains biens sont irremplaçables car ils n'ont pas d'équivalent objectif (une denrée) ou d'équivalent logique (un marteau). D'autres le sont en raison de leur unicité. Les reliques font partie de ces derniers. À partir du IV siècle, elles jouent un rôle central dans l'établissement et l'expression de la foi à travers le culte des saints. Les restes des saints
Le concile de Trente le réaffirme : « Si quelqu'un dit que dans le très Saint-Sacrement de l'Eucharistie ne sont pas contenus vraiment, réellement et substantiellement le corps et le sang en même temps que l'âme et la divinité de notre Seigneur Jésus Christ [...] mais dit qu'ils n'y sont qu'en tant que dans un signe ou en figure ou virtuellement : quili soit anathème » (session 14, 11 octobre 1551).

Le pain de l'hostie consommée ne « représente » pas le corps du Dieu incarné, il est sa chair.

Missel à l'usage du prieuré SaintMartin-des-Champs de Paris, 1408. (Paris, Bibliothèque Mazarine, ms 416, folio 219).

(Fig. 1) 
Le moine a qui appartenait ce petit ouvrage en ivoire de 10 centimètres priait devant l'image des instruments de la Passion du Christ (arma christi). Autant de reliques originales dont un grand nombre d'exemplaires étaient attestés à travers toute l'Europe.

Sur le verso de l'avant dernier panneau: les clous, le marteau, la pince, le bandeau, les 30 derniers (ici 29) donnés à Judas, et les empreintes ensanglantées ;

sur le recto du dernier panneau: le manteau, les dés, la lance, le roseau, le fléau, l'échelle, le tombeau (vide) vu d'en haut.

Livre de dévotion, $10,7 \times 6 \times 3,1 \mathrm{~cm}$, $200 \mathrm{gr}$, Westphalie (Allemagne), réalisé vers 1330-1350. (Londres, Victoria \& Albert Museum, numéro 11-1872).

(Fig.2).

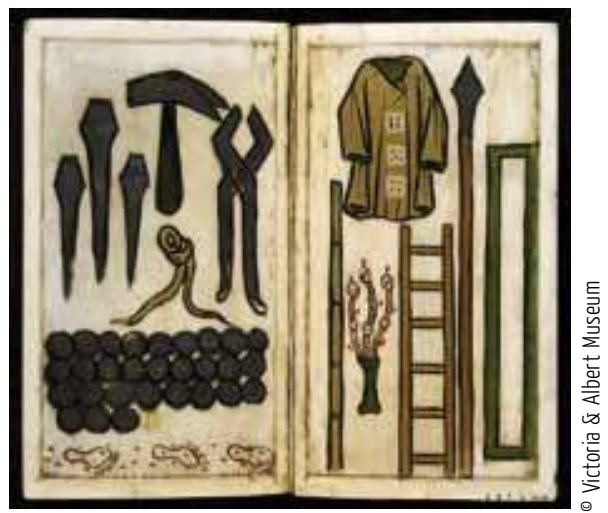

relient matériellement le fidèle à l'histoire sainte et à l'au-delà. Ils opèrent des miracles et sont en théorie indispensables à la consécration d'un lieu de culte dès le haut Moyen Âge (Méhu 2007). Or, comme le rappelle Jean Wirth, «l'unicité d'une relique est une condition sine qua non de son authenticité » (Wirth 2004 : 328). Une guerre des reliques était ainsi menée par les propriétaires. D'un côté, les doublons notoirement connus étaient déclarés frauduleux quand l'occasion se présentait. De l'autre, chacun certifiait l'authenticité de ses propres reliques au moyen de l'authentique (une bandelette sur laquelle était écrit le nom du saint ou de la sainte) et par une mise en spectacle de leur valeur intrinsèque : elles étaient à la fois montrées et protégées dans des reliquaires souvent précieux, ostensiblement scellés ou fermés à clé (Legner 1995). Généralement appliqué à un document, le terme « original » avait en latin le sens d'authentique et supposait que l'écrit émanât directement de son auteur, par opposition à copia qui signifiait l'abondance et copiare, reproduire. Cette distinction prend tout son sens dans le cas de la relique. Le morcellement permettait de contourner son caractère à la fois unique et indispensable dans la mesure où un seul fragment conservait la virtus de tout le corps. Mais le problème demeurait pour les reliques intégrales et les unicum. Ainsi, au début du xil siècle, Guibert de Nogent ${ }^{4}$ ironisait sur la bicéphalie de saint Jean-Baptiste dont la tête était conservée en même temps à Constantinople et à Saint-Jean-d'Angély.

Le paradoxe est plus flagrant encore, et en un sens parfaitement assumé, pour les reliques du Christ. En s'incarnant en l'homme, Dieu a laissé des traces de son passage et tout ce qui a été en contact avec lui, du fait même de son statut, possède littéralement une originalité. Mais la résurrection et l'ascension privent les fidèles du corps du Christ (tout comme l'assomption les prive du corps de la Vierge). Pour autant, comme si cela était «nécessaire », comme s'il ne pouvait pas en être autrement, le Moyen Âge se découvre des vestiges corporels de l'enfance du Christ et de la Passion: prépuce, dents de lait, sang et larmes viennent s'ajouter aux vestiges artefactuels (Figure 2). Si certaines reliques sont multiples, comme les clous du crucifiement ou les morceaux de la sainte croix - ou quantitatifs dans la mesure du vraisemblable - beaucoup sont indivisibles ou uniques, comme les traces de pas sur le Golgotha, théoriquement situées en un lieu précis, ou le linceul avec lequel Jésus essuya les pieds des apôtres (Jean 13, 5). À propos de ce linceul, Jean Calvin écrit dans le Traité des reliques (1543) : « Il y en a un à Rome à Saint-Jean de Latran, un autre à Aix en Allemagne, il faut bien que l'un ou l'autre soit faux ». L'impossibilité d'un double authentique et le surnuméraire étaient deux des principaux arguments de la critique du culte des saints: des bouts de la couronne d'épines ont dû être replantés pour reverdir ainsi partout! Pour le réformateur, on ne peut plus abuser du nom de Jésus « pour couvrir des fables tant froidement forgées $»$ :

Qu'ils [les catholiques] s'arment du nom de Constantin, ou du roi Louis [Louis IX], ou de quelque Pape: tout cela ne fait rien pour approuver que Jésus-Christ ait été crucifié avec quatorze clous: ou qu'on eût employé une haie tout entière à lui faire sa couronne d'épines: ou qu'un fer de lance en ait enfanté depuis trois autres [...] ou que d'un suaire seul, il en soit sorti une couvée, comme de poussins d'une poule (Calvin 1599 : 27-28, 36, 38).

Dans ce traité, véritable inventaire historique, Calvin ne dénombre pas moins de 117 reliques ou types de reliques ou lieux différents pour une même relique (sans compter 
les 100 lieux où l'on trouverait du sang christique), pour 44 reliques originales (robe pourpre, éponge... ) ou espèces de reliques (cheveux, deniers de Judas, ...) dont il conteste l'historicité (Calvin 1599 : 12-46). C'est en somme au prix de « tromperies honnêtes » (piae fraudes) que l'originalité et l'authenticité qui fondent la sacralité étaient sauvées. Des objets par définition ni échangeables, ni multipliables, ni fabricables ${ }^{5}$ se trouvaient, avec les contractions que l'on vient de voir, au cour du mythe et du culte chrétien ${ }^{6}$.

\section{L'objet rituel}

De très nombreux objets étaient impliqués dans les rites de la société médiévale, rites exceptionnels et prestigieux, comme le sacre d'un souverain, ou beaucoup plus courants mais non moins essentiels, comme la messe, l'ordination d'un prêtre ou l'entrée en vassalité 7 . C'est donc que les objets passaient pour des opérateurs indispensables, à côté de certaines paroles et de certains gestes effectués avec précision. Avant d'essayer de découvrir les objets qui ne pouvaient pas être supprimés ou remplacés sans faire échouer un rituel ou en empêcher l'organisation, décrivons le sacre royal français tel qu'il est censé se dérouler au $\mathrm{XIII}^{\mathrm{e}}$ siècle, selon des textes prescriptifs parfois mis en images. Quand elles existent, les images ne sont jamais seulement une illustration d'agrément, elles offrent une codification visuelle qui pouvait s'avérer pratique pour les contemporains eux-mêmes.

« On commence par préparer le trône (solium) au milieu du chœur » est la première phrase du livre du couronnement de Louis IX ${ }^{8}$. Le roi quitte la chambre et le palais pour l'église, lieu incontournable des opérations. Le seuil de l'église est franchi après avoir marqué une pause à la porte (hostium). Les sièges (sedes) autour de l'autel (altare) sont investis par les personnalités. Puis la sacro-sainte ampoule (ampulam) est conduite en procession avec croix et cierges (crubicus et cereis) jusqu'à l'autel sous un dais de soie (cortina serica) par des moines vêtus d'aube (albisindutis). Les promesses et les assentiments des parties ouvrent ensuite sur le rituel proprement dit. Sur l'autel sont posés la couronne royale (corona regia); l'épée (gladio) dans son fourreau; les éperons d'or (calcaribus aureis); le spectre doré (sceptro); la verge (virga) de justice, terminée par une main en ivoire (manum eburneam); les « chausses (caligis) de soie couleur d'hyacinthe

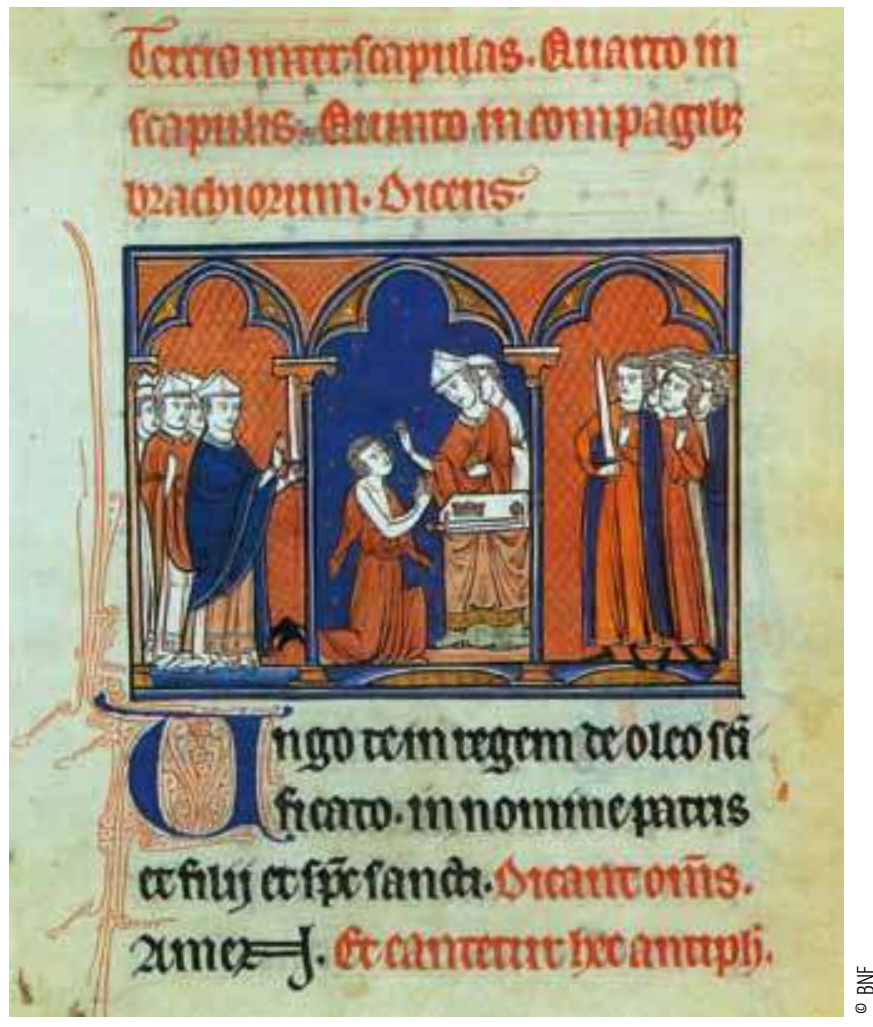

L'image suit le texte mais choisit ses moments: l'onction du roi, le rite de l'épée; le revêtement du roi, le soutien de la couronne, le baiser de paix. Ordo du couronnement de saint Louis, France, peu après 1254. (Paris, Bibliothèque nationale de France, ms Latin 1246, folios 17 et 26). (Fig. 3-4)

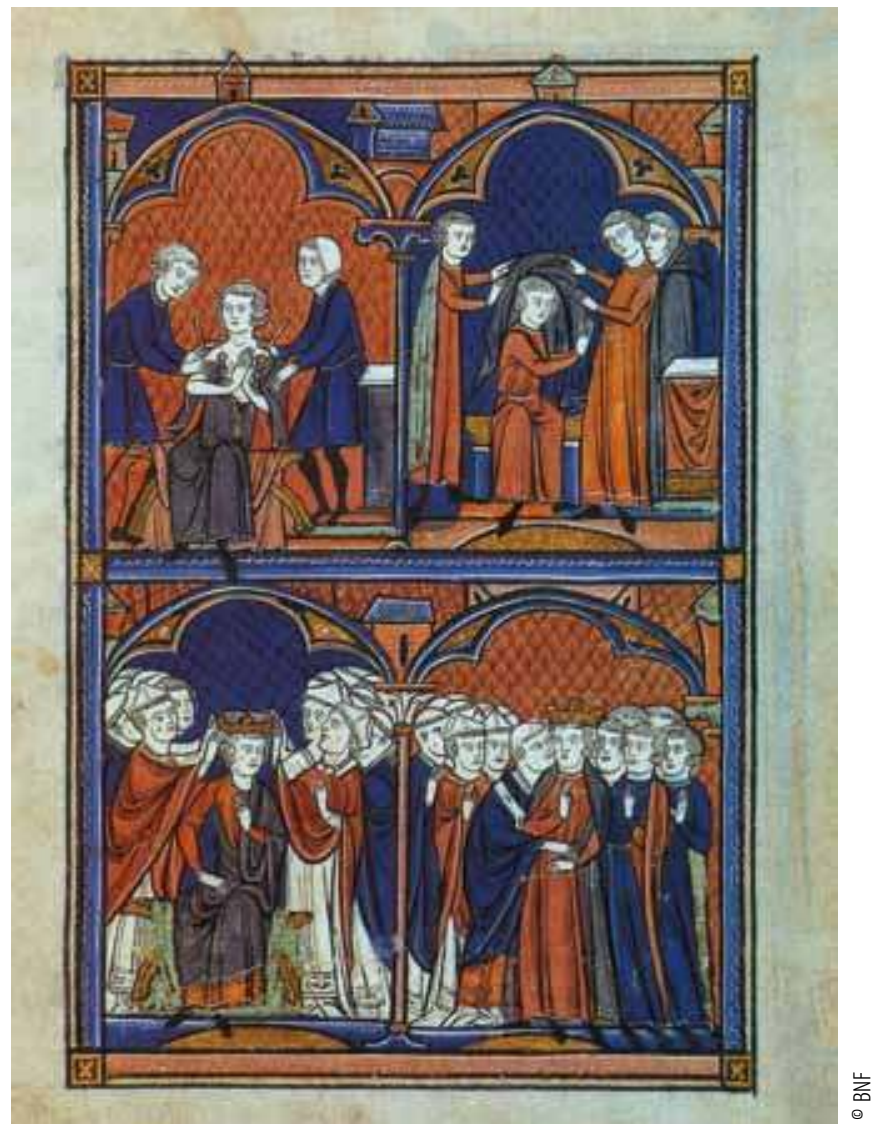


entièrement brodées de lis d'or, la tunique (tunica) de la même couleur et travaillée de même », confectionnée comme la tunique des sous-diacres à la messe; et enfin « le surcot (socco) aussi de la même couleur et travaillé de même », à la façon d'une « chape (cape) de soie sans capuchon (caparone) ». Les vêtements que le roi porte sont alors déposés par lui, sauf sa tunique. La tunique est ouverte sur le devant et dans le dos, des attaches en argent (ansulis argentes) la maintiennent aux épaules. Puis les chausses sont enfilées aux jambes du roi par le grand camérier. Les éperons, signe de son état de chevalier, sont noués puis tout de suite enlevés par le duc de Bourgogne. L'épée, insigne du gouvernement du royaume, est ceinte aux hanches du roi et tirée du fourreau par l'archevêque. Le fourreau (vagina) est déposé sur l'autel et l'épée remise au roi. Portée par le roi sur l'autel, l'épée lui est ensuite redonnée par l'archevêque; le roi la donne sans attendre au sénéchal. Nue et droite, elle doit être portée par ce dernier jusqu'à la fin de la cérémonie (Figure 3). La tunique du roi, qui s'est agenouillé, est alors dégrafée tandis qu'une aiguille d'or (acu aurea) est trempée dans l'huile céleste de la sainte ampoule et sert à mélanger l'huile au chrême (crismate) préparé sur l'autel dans une patène (patenam). Laiguille, tenue par l'archevêque, oint alors le corps du roi en plusieurs endroits, d'abord le front, puis la poitrine. Les attaches sont refermées et la tunique violette et le surcot enfilés l'un sur l'autre au roi par le grand camérier, de telle sorte que l'ouverture du surcot laisse libre la main droite et que le côté gauche soit relevé comme une chasuble (précisions données par le texte). Le sceptre est donné au roi dans sa main droite, la verge dans sa main gauche par l'archevêque, et la couronne est posée par ce dernier sur la tête du roi, avant d'être touchée et soutenue par tous les pairs de France, clercs et laïcs (Figure 4). Le trône paré de soie attend désormais le roi, conduit par l'archevêque. Le siège élevé sur lequel il prend place permet de montrer à tous le roi couronné. La mitre ayant été déposée par l'archevêque en signe de révérence, ce dernier et tous ceux qui tiennent la couronne donnent un baiser au roi avant que l'épée et d'autres objets, bracelets, pallium, anneau, lui soient remis par les évêques ainsi que le sceptre, la verge et la couronne à nouveau. La remise de ces insignes est associée à des paroles qui décrivent leurs actions sur la personne du roi et ce qu'un roi doit faire pour la bonne conduite de son royaume. Alors, le roi, la couronne sur la tête, revient de l'autel pour s'asseoir à nouveau sur le trône qu'il a quitté pour la remise des insignes. Là, il écoute sa confirmation et donne le baiser de paix. Les cloches se mettent à sonner, retentissent. La messe achevée, la couronne cérémonielle est échangée par une couronne plus pratique, et le roi rentre publiquement au palais, devancé par l'épée toujours au clair.

Les paroles redoublent l'action des objets. Larchevêque du sacre royal dit: «par cette onction, je te fais roi », « reçois l'anneau » qui est le signe (signaculum) que « tu es ordonné tête et prince du peuple ». Addition faite, il faut 34 objets et substances pour faire un roi, à peu près autant pour faire un évêque suivant le manuel de la curie romaine, et ce nombre diminue quand on descend dans la hiérarchie (un prêtre requiert une mitre, dans la mesure où l'évêque doit s'en décoiffer, et un surplis qu'endosse l'impétrant, si l'on exclut sa tonsure) $^{9}$. Mais quels objets étaient vécus comme indispensables, faisaient « ce que ni les gestes ni les mots ne suffisent à faire » ? La sainte ampoule qui marque le début du rituel royal et la couronne sont sans doute ce que Pierre Lemonnier (2012) appelle des « objets stratégiques », auxquels on peut ajouter l'épée, présence stratégique, compte tenu des choix faits par l'enlumineur (Figures 3-4). Mais, pour la consécration du pape, le défaut d'ombrelle (umbraculum), qu'un valet devait théoriquement tenir au-dessus de la tête du pape au moment de la procession, n'était évidemment pas rédhibitoire à sa consécration ${ }^{10}$. 
Jacques Le Goff a montré que la nécessité se trouvait surtout dans la cohérence et la succession des phases essentielles. Dans son étude sur le rituel de la vassalité (1999 [1976]), il note que dans les récits qui en sont faits, les trois actions rituelles (hommage, foi, serment) sont très souvent exprimées en une seule phrase, fortement marquée par des conjonctions de coordination (1999 : 348). L'élément irremplaçable était moins dans la lettre que dans le respect d'un système logique (« [le rituel] ne fonctionne que si aucun élément essentiel n'y manque ») et dans la signification symbolique que l'on attendait de chaque phase. Non pas telle formule, mais, dans la phrase d'hommage, l'idée de subordination. Non pas tel geste, mais, par les mains du seigneur entourant celles de son vassal, l'idée de protection. Non pas tel objet, mais une matérialisation de la terre sous une forme ou une autre (motte, gazon, fétu).

Quelques témoignages incitent néanmoins à penser que certains objets étaient symboliquement incontournables pour la réalisation de certains rituels ecclésiastiques. Un volumen composé autour de 969 pour l'évêque Landulf de Bénévent propose une image de chaque rituel d'ordination (Reynolds 1983; Palazzo 1999 : 85-87). Pour chaque genre ou degré de clerc, Landulf fit peindre le moment du don de l'objet emblématique de la charge. L'ostiaire, chargé de

la porte de l'église, reçoit les clefs; le lecteur, le «livre de prophètes », et ainsi de suite. Le sacramentaire de Marmoutier, réalisé au milieu du Ix siècle, s'ouvrait déjà sur une pleine page figurant cette fois les rituels de manière synthétique (Figure 5). Si l'on poursuit de gauche à droite: le sous-diacre, au centre du registre inférieur, tient une patène et un calice qu'il reçoit selon l'ordo de la main de l'évêque. On donne à l'exorciste un livret (libellum) où sont inscrits les exorcismes, et à l'acolyte un chandelier « avec le cierge éteint ». L'imposition des mains et la remise des objets de culte étaient déjà dans l'Église primitive les deux moments essentiels de l'investiture ${ }^{11}$.

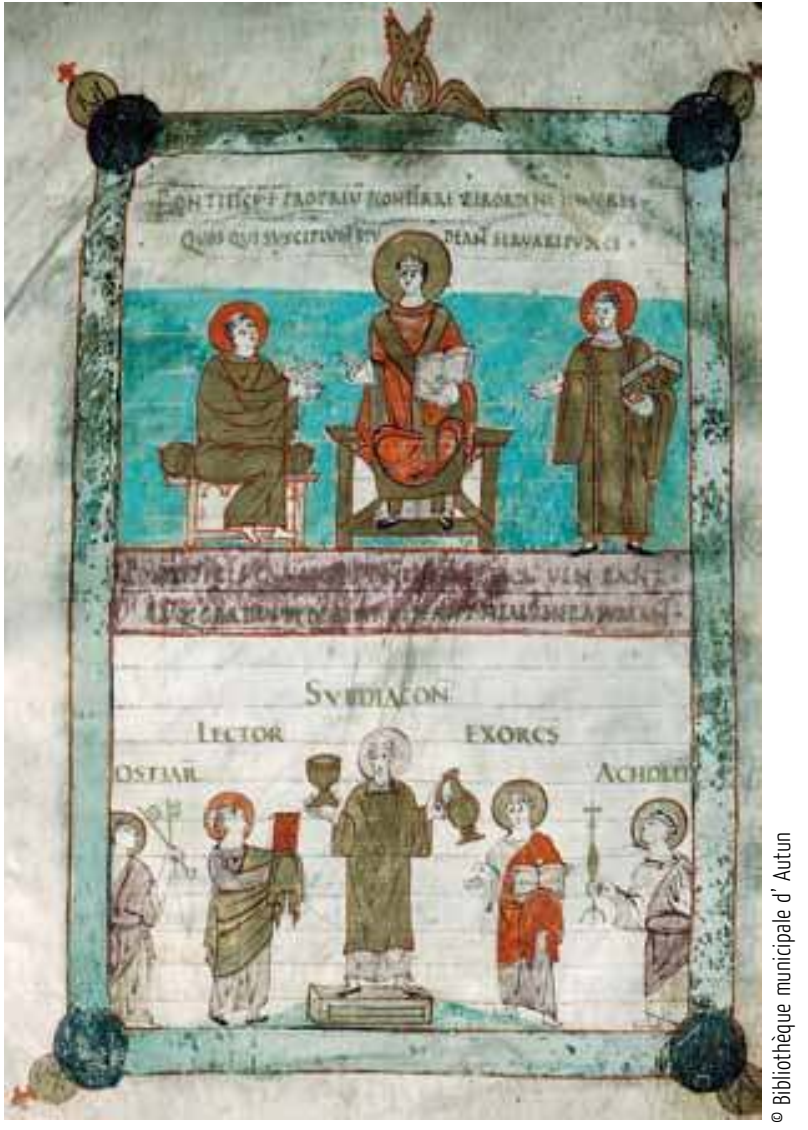

Au registre inférieur de l'image, le rituel d'investiture des ordres mineurs est figuré de manière synthétique. Sans doute à la demande de l'évêque, présent dans la partie supérieure de l'image entre le prêtre et le diacre portant un livre, le peintre a représenté chaque clerc avec le symbole de sa fonction, quili reçoit lors de son investiture. Sacramentaire de Marmoutier à l'usage d'Autun, France (Ouest), vers 845-850.

(Autun, Bibliothèque municipale, ms 19bis, folio 1 verso).

(Fig. 5)

\section{Cas pratiques}

L'archevêque de Rouen Eudes Rigaud nous a laissé un témoignage exceptionnel de ses visites pastorales. Il raconte presque au jour le jour la situation des abbayes et des paroisses qui se trouvent sous son autorité, consignant scrupuleusement les déviances, le manque de matériel, les injonctions qu'il a adressées à ses hôtes. 
En 1252, à Eu, Eudes Rigaud constate que les moines n'ont toujours pas récupéré un calice d'or, élément clé de la vie liturgique, et il ordonne qu'ils somment le vicomte de le leur rendre sous peine d'excommunication ${ }^{12}$. Au prieuré de Beaulieu, en 1269, Eudes note que les chanoines ne possèdent pas assez de calices et ordonne d'y remédier ${ }^{13}$. Ailleurs, il relève l'absence de nappes d'autel et d'ornements, et semble effaré par l'état pitoyable du reliquaire ${ }^{14}$. À plusieurs reprises dans son Registre, l'archevêque déplore le manque de livres, dont certains sont utilisés pour l'office. Au cours d'un séjour à l'église de Saint-Mellon de Pontoise, le chapelain Thomas lui apprend qu'il célèbre la messe quotidiennement dans un couvent proche mais qu'il ne la fait que « rarement ou jamais » à Saint-Mellon, par manque de missel. Refus donc, plus qu'incapacité du chanoine. Refus également, mais cette fois d'Eudes, quand il apprend qu'une nonne assiste le prêtre pour la messe à Saint-Saëns en $1253^{15}$ ou que les clercs portent des vêtements de laïcs. Les interdits portent donc également sur la présence de certains éléments dans la mesure où l'on estime qu'ils empêchent le bon déroulement des rites et plus largement de la vie claustrale ou canoniale: leur suppression est jugée indispensable. Ainsi, le Synodal de l'Ouest, un de ces documents qui compilaient les décisions promulguées par le clergé diocésain que chaque prêtre devait idéalement consigner dans un livre, interdisaient les meubles personnels dans l'église ${ }^{16}$.

Ces «statuts » nous livrent quantité de situations où le rituel est menacé, voire interdit, ainsi que les procédures pour y remédier. Il est défendu au prêtre de célébrer la messe sans chaussure (sine caligis) ${ }^{17}$ et aucun clerc n'est autorisé à servir à l'autel s'il ne porte pas un surplis ou une chape fermée ${ }^{18}$. Le respect des substances sacrées et du matériel qui se trouve dans la maison de Dieu est une condition implicite à tout acte liturgique. Selon les termes de ces textes, la vétusté pouvant induire à l'irrévérence, il faut renouveler l'eau bénite, l'huile, le chrême, la sainte eucharistie tous les huit jours. Les burettes qui servent à l'autel doivent être propres et sans fêlures ${ }^{19}$ et le vin ne doit être ni piquant ni moisi ${ }^{20}$. Enfin, on incite les paroissiens à pourvoir l'église de tous les objets qui y manqueraient, des aubes et des nappes en particulier ${ }^{21}$.

Les incidents qui peuvent survenir pendant un rituel sont particulièrement éclairants. Lofficiant qui consacrait avec du vin, mais sans eau, ou (plus grave) avec uniquement de l'eau, devait être puni. Et «s'il arrivait que par la suite d'une négligence, la lecture du canon et la consécration achevée, il ne se trouve ni vin ni eau dans le calice, le prêtre doit aussitôt verser l'un et l'autre et recommencer » l'opération prudemment au point où il s'est arrêtée ${ }^{22}$. $\mathrm{Ou}$ :

Si une mouche ou une araignée ou quelque chose de tel est tombé dans le calice qui ne puisse être absorbé sans provoquer de vomissement ou sans danger pour le corps, que ce soit brûlé dans le feu et qu'on consomme le sang du Seigneur. Mais on doit en premier lieu répandre du vin dans le calice et le laver le plus précautionneusement et le plus rapidement qu'il se pourra, et ensuite brûler ce qui est tombé de dedans au-dessus de la piscine, et que cette ablution soit prise par le prêtre $[\ldots]^{23}$.

La pureté est presque toujours en jeu. Lorsqu'il célèbre la messe, le prêtre doit avoir un manuterge à côté de l'autel, c'est-à-dire un petit linge pour ne pas s'essuyer la bouche et le nez à ses vêtements. Après la messe, le calice doit être enveloppé dans une étoffe très propre/pure (panno mundissimo) qui ne sert qu'à cela ${ }^{24}$. Pour la même raison, les linges d'autel ainsi que les vêtements rituels doivent être lavés par quelqu'un d'honnête et « sans mélange avec d'autres linges » (sine appositione aliorum pannorum) ${ }^{25}$. 
Dans le cadre rituel, si certains objets ne pouvaient manquer ou devaient présenter certaines qualités matérielles, ils devaient de toute façon être bénis au préalable, autrement dit changer de statut - sortir du circuit des usages ordinaires - pour pouvoir être employés. Cette opération routinière mais inconditionnelle concernait la plupart des objets de la vie religieuse. Une fois béni, l'objet n'était plus comme son équivalent non béni. Sa « qualité » était désormais autre. Le Pontifical de la curie romaine qui réunit les ordines (rituels) de l'évêque, prévoit treize rituels de bénédiction d'objets à côté des bénédictions de personnes et d'aliments ${ }^{26}$. Bénédiction de la besace et du bâton de pèlerins, de la première pierre d'une maison, de la première pierre d'une église, d'une nouvelle maison, de l'église elle-même, de l'autel portatif, de la patène, du calice, des corporaux, c'est-à-dire des linges d'autel, d'une nouvelle croix, de l'encens, des vêtements sacerdotaux (chasubles, dalmatiques, étoles, ...) et de la cloche (Figure 6).

Ainsi, pour la cloche, on dira tout d'abord les psaumes de la pénitence « [...] Écoute Seigneur, réponds-moi. Des profondeurs, je crie vers toi » et d'autres oraisons:

Seigneur, bénis cette eau de ta bénédiction céleste, et que la puissance de l'Esprit [virtus spiritus sancti] descende sur elle, afin que, lorsque cet humble objet prévu pour appeler les fils de l'Église y aura été plongé, il puisse chaque fois que retentira sa sonnerie chasser au loin la force des ennemis, l'ombre des spectres, l'assaut des ouragans, les ravages de la foudre [...]. Et quand les fils des chrétiens auront entendu ses coups, que grandisse en eux l'ardeur de la dévotion [...].

Puis on versera du sel dans de l'eau bénite (l'exorcisme et la bénédiction de l'eau et du sel ayant déjà été réalisés) pour que le mélange devienne un «sacrement salutaire ». Suivra une oraison et la cloche sera lavée; on chantera à nouveau pendant que l'officiant signera la cloche sur l'extérieur avec de l'eau bénite et de l'huile sainte, en y dessinant une croix ${ }^{27}$. Enfin, on hissera la cloche. La cloche non bénie sonnera, mais la cloche bénie repoussera le diable et les tempêtes, sa sacralité sonore résonnant dans la campagne alentour.

Des objets créés ou employés à la seule fin d'un rituel, on est passé au rituel qui fait de certains objets des objets à part, aptes à des usages exclusifs, doués d'une force que n'ont pas leurs semblables. Rendus uniques pour les circonstances, d'autres objets l'étaient encore «par» les circonstances. En effet, beaucoup des objets qui dans la société médiévale prennent place dans une opération rituelle étaient plus ou moins longtemps détournés de leur utilité et de leur sens habituel, pour acquérir une identité singulière. Comme le souligne Laurent Feller, l'objet transmis par le seigneur au vassal lors de l'investiture d'une terre (un gant, un bâton, une bannière) « sert de support au droit exercé sur la terre et se dégage de sa fonction première ». L'épée cessera « d'être seulement une arme pour devenir un signe de la possession. Exhibée dans un procès, elle prouve l'existence de l'investiture, jusqu'au moment, où, la cérémonie fondatrice ayant été oubliée, elle redevient une arme et apparaît comme une menace aux juges ayant 
à [instruire] l'affaire » (Feller 2010 : § 3). Transformé par une opération magique ou pris à partie dans une institution, l'objet ne peut plus être remplacé par son semblable.

\section{Le « produit » économique}

La longueur du drap est contrôlée par les ouvriers et les inspecteurs. Ils vérifient que la pièce corresponde à son type légal, c'est-àdire à une qualité donnée.

Témoignage plus précoce que les textes. Lettre ornée des Morales sur Job, vers 1120.

(Dijon, Bibliothèque municipale, ms 173, folio 92 verso).

(Fig. 7)

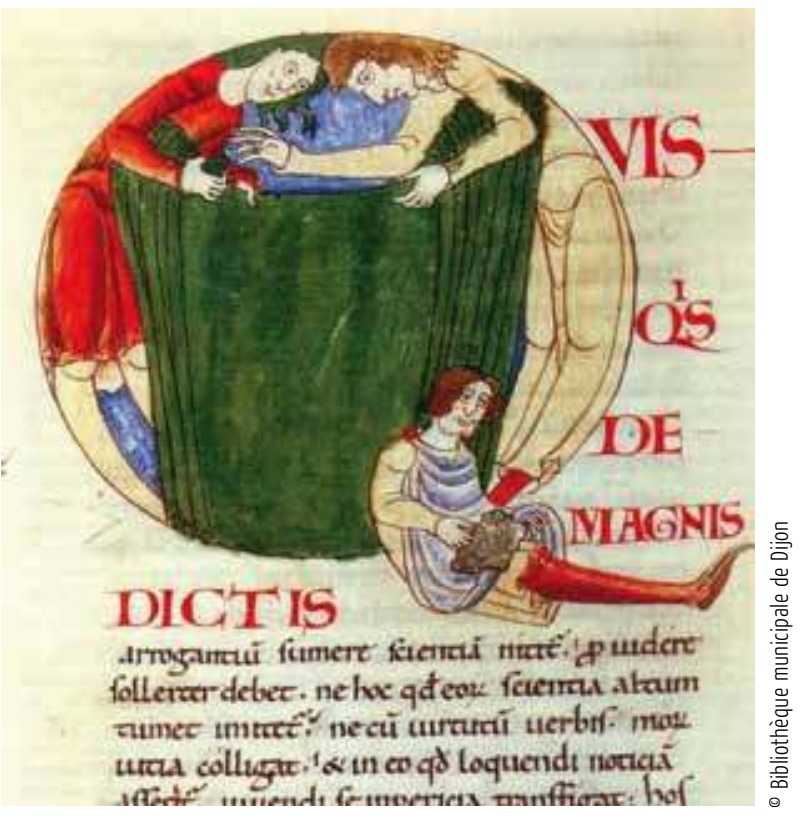

L'impossible substitution entre deux objets d'une même classe touche l'économie médiévale à partir des XII ${ }^{e}$-XIII ${ }^{e}$ siècles. Un «drap de Frise » n'est pas un «drap d'Ypres ». Nous connaissons bien ce mode d'existence des objets: une Porsche n'est pas une Ferrari, bien qu'elles remplissent les mêmes fonctions de déplacement et de distinction sociale; un Bordeaux n'est pas un Bourgogne, même s'ils répondent tous les deux à la vertu inébriante du vin. Nous sommes habitués aux biens à forte " personnalité », reconnus au premier coup d'œil ou par des traits identificateurs: nom, emballage, certification, un ensemble de signes extérieurs auxquels l'acheteur accorde une confiance suffisante. Ces biens tirent une part de leur valeur de leur identité même, d'une relation « fétichiste » fondée sur la réputation et le capital social qu'il contient.

C'est à la fin du Moyen Âge que ce régime référentiel de production et de consommation émerge. Généralement associée à l'économie moderne, au plus tôt à la période classique (Grenier 1996), la production d'objets hautement standardisés et marqués, dont le type possède une existence durable sur les marchés - ce que j'appelle un «produit »-se développe lentement à la suite d'une série de facteurs structurels que connaît l'Europe dès le $\mathrm{xI}^{\mathrm{e}}$ siècle. Notamment l'urbanisation, qui entraîne la division du travail et la spécialisation de l'artisanat, et l'augmentation du nombre de personnes susceptibles de consommer des biens de luxe ou fabriqués en dehors de l'espace domestique.

La non-substitution symbolique des biens manufacturés est précoce dans le secteur du textile. Il est aussi le mieux documenté pour comprendre le changement qui touche un grand nombre de biens dans les siècles suivants.

Si l'on considère le drap de la fabrication à la vente, on constate que le phénomène est présent à tous les niveaux où le produit est singularisé. Un document de 1282 réglementait pour Bruxelles trois longueurs différentes (48, 42 et 37 aunes et demi) et ces longueurs reflétaient une hiérarchie relative entre les types d'étoffes; la qualité intermédiaire était ainsi appelée di lange lakene van XLII (tweeënveertig) ellen (Favresse 1961 : 98). La « loyauté » ou la «bonté » du drap dépendait par conséquent de ce critère, et le mesurage était une des nombreuses vérifications techniques effectuées par les jurés pour autoriser sa mise sur le marché (Figure 7). Le drap qui présentait des défauts était déclaré «faux » et il pouvait être coupé en deux ou trois pièces ou vendu à la coupe pour ne pas être écoulé au prix du type auquel il était censé appartenir. Aux caractéristiques propres à l'objet (longueur, armure, rendu) s'ajoutaient des caractéristiques extérieures dont le but - certifier et pouvoir identifier la pièce - était en pratique 
d'empêcher la copie et la vente trompeuse. Les lisières, des fils de couleurs placés sur les bords longs du drap, permettaient « d'avoir congnoissance que ce soit draperie faite [par exemple] d'aignelins ». Le nombre et la teinte des fils, sorte de code-barres avant la lettre, étaient légalement fixés « afin que l'on puist perchevoir et avoir congoissance que les dis draps aront esté fais [en l'occurrence] en la ville d'Arras ${ }^{28} \gg$. Le pliage était lui aussi caractéristique d'un centre de production ou d'une espèce. Le sceau, en particulier, fixé dans l'étoffe par un système de rivet par les inspecteurs assermentés (eswardeurs), constituait la marque d'authenticité et le signe d'identité les plus forts. Avec là encore le même double objectif: " autentiqués loyaument " les pièces et leur servir de « seignet de descongnissance [de reconnaissance] ${ }^{29}$ \%. Enfin, le nom à lui seul créait de la différence. Le tarif de péage dit de $1296^{30}$ établi pour les marchandises entrant dans Paris nomme pas moins de 57 villes ou provenances: " petit drap de Bresselles, II sous, royés [rayés] de Tournay VI denier la pièce », etc.

Assez logiquement, c'est par l'appellation prestigieuse et la désinformation que les marchands peu scrupuleux vendaient un drap médiocre à leur avantage, comme en 1380, dans des boutiques volontairement obscures (« escuras e poca lunbre ») où, par exemple, "pour du Malines» (por Melinas) ils montrent « du Rouen » ${ }^{31}$. L'expression « un payno por otro », un « drap pour un autre », que l'on trouve dans les amendements de 1330 des privilèges de Navarre de $1237^{32}$, traduit parfaitement l'idée de substitution. La substitution est interdite mais elle est d'autant plus tentante et réalisable qu'il y a de signes pour tenter d'y remédier. Car c'est bien à la construction d'un modèle économique par réaction à ses propres conséquences indésirables à laquelle on assiste. Plus le produit était imité, plus il était rendu « unique», et plus il était singularisé, plus il pouvait être imité. Le fraudeur s'emparait de tout ce qui faisait qu'un drap n'en était pas un autre. Non seulement l'apparence était imitée (comme pour la fausse monnaie et les fausses mesures), mais aussi les marques d'authenticité (comme pour le marché des reliques). Les sceaux des meilleurs draps étaient souvent récupérés pour être placés sur d'autres draps, et vers 1282 un drapier est condamné à Valenciennes par le prévôt de la halle pour avoir «bullé [scellé] ses draps de fausses bulles $»^{33}$.

Pourquoi la réplique d'une étoffe ne valait-elle pas l'occurrence du type imité? Pour la relique, il s'agit de l'originalité. Pour l'irremplaçabilité économique, deux critères émergent de la documentation législative (règlements de métiers) et judiciaire (plaintes et jugements). Il s'agit de la qualité et, plus surprenant peut-être, de la propriété. Une qualité donnée était le résultat d'une « technologie » collective locale, d'une concentration de spécialistes, de moyens, de connaissances et d'accords politiques aussi. Chaque pièce était l'alter ego satisfaisant d'une autre, en vertu d'un schème de fabrication préétabli, contrôlé à différentes étapes, validé en bout de course par des marques officielles qui garantissaient l'identité des pièces. Étrangeté vertigineuse, mais que nous ne ressentons plus, d'être totalement indifférent aux occurrences, pour ne nous soucier que du type, « du modèle » disons-nous. Face aux contrefacteurs qui ne peuvent (ou ne veulent) sans doute pas atteindre la qualité des originaux, les producteurs de Saint-Omer déclarent que leur draperie « est molt diffamée » ${ }^{34}$. Mais il y a plus. Contre les duplicata, les fabricants invoquent l'idée de propriété économique et auctoriale, une sorte de copyright ou de brevet ${ }^{35}$. Même si une imitation pouvait être parfaite, de sorte qu'elle pût se ranger dans la pile des originaux, les producteurs opposaient aux imitateurs organisés l'argument de la propriété du type qu'ils produisaient: si une pièce n'était pas réalisée dans son centre d'origine, elle était d'emblée déclarée « fausse » par le législateur. 
En somme, dans ce cas-ci, le problème ne porte pas sur l'unicité mais sur l'identité, au double sens de mêmeté et d'identitaire.

En reprenant maintenant les différents cas de figure - depuis le phénomène de la représentation sociale jusqu'au régime économique de type référentiel qui s'empare de l'univers matériel médiéval - nous pouvons établir le schéma suivant:

\begin{tabular}{|c|c|c|c|}
\hline $\begin{array}{l}\text { Représentation } \\
\text { (égalité relative) }\end{array}$ & $\begin{array}{l}\text { Produit } \\
\text { (identité) }\end{array}$ & $\begin{array}{l}\text { Relique } \\
\text { (unicité) }\end{array}$ & $\begin{array}{l}\text { Objet rituel } \\
\text { (transformation) }\end{array}$ \\
\hline$A \geq A$ & $\overbrace{A^{\prime}=A^{\prime}=A^{\prime} \ldots}^{A}$ & $\begin{array}{c}A \\
\neq \\
A^{\prime} \neq A^{\prime} \neq A^{\prime} \ldots\end{array}$ & $\begin{array}{l}A^{\prime} \\
A^{\prime} \\
A^{\prime}\end{array}$ \\
\hline $\begin{array}{l}\text { Difference de principe } \\
\text { ou de probleme: } \\
\text { Connaissance }\end{array}$ & Confusion & Vérité & Propriétés \\
\hline
\end{tabular}

La culture médiévale, à l'égard des reliques, parvient à dépasser la contradiction unique-multiple malgré ses incessants rappels, et ce n'est pas chose si « naturelle » puisque les protestants y mettent un terme pour leur confession. Le christianisme médiéval est du reste familier de ce genre d'antithèse. À commencer par le dogme de La Trinité: un Dieu unique en trois hypostases (le père, le fils et le saint-esprit), c'est-à-dire « l'unité de la distinction en Dieu » pour reprendre la formule de Denys l'Aréopagite ${ }^{36}$. Une ambivalence structurelle existe également à propos de l'imitation et de la ressemblance dans le fait qu'elles sont condamnées sur le plan économique (la contrefaçon) et en politique (dans le cas de l'imposture), mais que l'homme a été fait à l'image et à la ressemblance de Dieu ${ }^{37}$ et que le grand modèle chrétien de comportement est l'imitatio. Il faut imiter le Christ et les saints - une maxime que Thomas a Kempis choisit pour le titre de son ouvrage de dévotion, le De imitatione Christi au début du Xv siècle, au moment où les industriels sont plus que jamais aux prises avec le démon de l'imitation. Plus proche encore de l'activité technique, pour les penseurs du Moyen Âge ${ }^{38}$, l'acte de fabrication idéal, la manière propre à l'homme d'agir sur la matière n'est pas d'inventer ou d'être original, mais, suivant l'opinion de Platon et d'Aristote, d'imiter la Nature. 


\section{NOTES}

Photo d'ouverture : Le moine a qui appartenait ce petit ouvrage en ivoire de 10 centimètres priait devant l'image des instruments de la Passion du Christ (arma christi). Autant de reliques originales dont un grand nombre d'exemplaires étaient attestés à travers toute l'Europe. Sur le verso de l'avant dernier panneau : les clous, le marteau, la pince, le bandeau, les 30 derniers (ici 29) donnés à Judas, et les empreintes ensanglantées ; sur le recto du dernier panneau: le manteau, les dés, la lance, le roseau, le fléau, l'échelle, le tombeau (vide) vu d'en haut. Livre de dévotion, 10,7 x 6 x 3,1 cm, 200 gr, Westphalie (Allemagne), réalisé vers 1330-1350. (Londres, Victoria E Albert Museum, numéro 11- 1872).

1. Je remercie Pierre Lemonnier, Christian Bromberger et Pierre-Olivier Dittmar pour leurs suggestions lors du colloque Irremplaçables? Approches archéologiques et anthropologiques d'objets uniques, précieux, nouveaux organisé par Maxence Bailly, Pierre Lemonnier et Sandra Revolon, les 23 et 24 mai 2011 à l'Université d’Aix-Marseille. La seconde partie a également bénéficié d'une communication sur un thème proche: «Pas de copie sans modèle. L'imitation, une nouvelle fraude économique au xiII ${ }^{\mathrm{e}}$ siècle », présentée le 16 septembre 2010 au colloque international organisé à l'École des hautes études en sciences sociales par Corneliu Dragomirescu, Éric Hold et Jean-Claude Schmitt sur La substitution. Sens et pratiques dans la pré-modernité.

2. Ici et plus loin j'ai principalement consulté pour l'ancien français: Rey 1998, Godefroy 1881-1902 et Tobler, Lommatzsch \& Helmut 1925-2002; pour le latin médiéval: Du Cange 1883-1886 et Blaise 1975.

3. Æneas Sylvius Piccolomini (Pie II), Commentarii rerum memorabilium quae temporibus suis contigerunt, livre VII, (éd.) A. van Heck, Vatican, Biblioteca apostolica Vaticana, 1984 : 185.

4. Guibert de Nogent, De sanctis et eorum pigneribus, (éd.) R. B. C. Huygens, Turnhout, Brepols (Corpus christianorum. Continuatio, vol. 127), p. 102. Voir Platelle, 1999 et Duby, 1996 : 334 et sqq.

5. À l'exception peut-être du vin transsubstantié en sang du Christ qui tombe sur le corporal, la nappe d'autel, la chasuble ou l'aube: si cela arrivait, expliquent entre autres les Statuts synodaux de Paris, « la partie touchée sera [...] découpée et conservée comme relique ». Les statuts synodaux français du XIII ${ }^{e}$ siècle, (éd.) O. Pontal, Paris, Bibliothèque nationale, 1 , p. 81, § 75.

6. De manière éclairante, Thierry Lenain $(2008,2011)$ fait de la « critique d'authenticité » des reliques médiévales l'origine de la problématique du faux en art. Je le remercie de m'avoir fourni le manuscrit de Art forgery désormais paru (Lenain 2011).

7. Du Cange dresse une liste de 100 objets à l'article «Investitura» du Glossarium, op. cit., t. 3, col. 1520-1538.

8. Ordo ad consercrandum et coronandum regem, Paris, Bibliothèque nationale, ms latin 1246, folio 1-42, spécialement les folios 4, 15 verso, 17, 19, 26, 26 verso, 27 verso, 28, 29, 37 verso et 42. Sans doute réalisé vers 1254 et d'un usage liturgique incertain, cet ordo vaut a priori pour la seconde moitié du xiII ${ }^{\mathrm{e}}$ siècle, entre l'ordo de l'« ordinaire de Reims » pour la période précédente et le « dernier ordo capétien » pour l'après saint Louis. cf. l'étude de Le Goff \& al. 2001.

9. Pontificale secundum consuetudinem et usum romane curie, I, § 1-3, p. 34 (ordination du clerc), XI, § 9-12, 18-21, 23 et 28, p. 72 et s. (consécration de l'évêque élu), éd. M. Goullet, G. Lobrichon \&É. Palazzo, Le Pontificale de la curie romaine au XIII ${ }^{e}$ siècle, Paris: Cerf, 2004.

10. Ibid., XIII B, § 34, p. 112.

11. Hippolyte de Rome, Tradition apostolique, 4-13, 19 passim, éd. Sources chrétiennes, vol. 11, p. 46 et s. Constitutions apostoliques, livre VIII, 19-22, éd. Sources chrétiennes, vol. 320. Statuta Ecclesiae antiqua, canons 90-102, éd. Corpus christianorum. Series latina, vol. 148, p. 181-185.

12. Eudes Rigaud, Regestrum visitationum, (éd.) Th Bonnin, Rouen, 1852, à la date du 18 mai. Sur ce document, voir Andrieu-Guitrancourt 1938.

13. Eudes Rigaud, Regestrum visitationum, op. cit., p. 632.

14. Le 28 août 1258. Ibid., p. 315-316: « Non habebant pallas altarium nec ornamenta sufficienter; precepimus hec emendari. Capse in quibus dicuntur requiescere corpora quoumdam Sanctorum et Sancti Melloni, adeo putrefacte erant et vetustae consumpte, quod vix poterant manu contingi; precepimus hoc emendari...»

15. Ibid., p. 187.

16. Synodal de l'Ouest, § 24. Les statuts synodaux, op. cit., t. 1, p. 155.

17. Statuts de Paris, § 91, ibid., t. 1, p. 87.

18. Ibid., § 22, p. 61 .

19. Ibid., \& 18, p. 58.

20. Statuts de Bordeaux (1234), § 30, Les statuts synodaux, op. cit., t. 2, p. 58-59.

21. Statuts de Paris, § 93, ibid., t. 1, p. 87.

22. Ibid., § 74, p. 79. Version proche dans les Statuts de Bordeaux, \& 30, ibid., t. 2, p. 58-59.

23. Statuts de Paris, $\S 76$, Les statuts synodaux, op. cit., t. 1 , p. 80-81. Dans une version proche dans les Statuts de Bordeaux, § 32 : «Item s'il arrivait par hasard qu'une 
araignée, une mouche ou quelque reptile tombe dans le sacrement du sang... », ibid., t. 2, p. 60-61.

24. Synodal de l'Ouest, § 13, ibid., t. 1, p. 149.

25. Ibid., \& 22, p. 153. Invoquant le canon 19 du concile de Latran IV.

26. Pontifical, supra.

27. Ibid., chap. XV, p. 243.

28. Ordonnance générale sur la draperie par Philippe le Hardi en mai 1394 à Neuchâtel, § 7 et 6 . Documents publiés par Espinas 1923, t. 2: 948. Pour cette partie, les volumes du Recueil de documents relatifs à l'histoire de l'industrie drapière en Flandre d'Espinas \& Pirenne (1906-1966) ont été principalement consultés.

29. Espinas 1923, t. 2, respectivement: 365 et 364.

30. Ordonnance des coustumes de Paris, combien que chascune marchandise doit paier de peage éditée par L. Douët d'Arcq, Revue archéologique, 9, 1852 : 213228. Elle date plutôt de 1337, cf. Bauthier 1966.

31. Récit de Pero López de Ayala, cité par M. Grande Esteban, Introducción a Rimado de Palacio, Madrid: Emiliano Escolar editor, 1981.

32. Fuero General de Navarra, Amenjoramiento, chap. XVI, éd. Pablo Ilarregui, Segundo Lapuerta, Pampelune: Imprenta Provincial, p. 151.

33. Cité avec d'autres cas par Cardon 1999 : 598.
34. Pièces et cas décrits par Espinas 1923, t. 1: 100 et t. 2 : 796-797. De nombreux exemples réunis par Abraham-Thisse 2006.

35. Sur cette législation et les marques de commerce, Espinas 1923, t. $1: 98-149$ et t. 2:348-407.

36. Denys l'Aréopagite (début $\mathrm{VI}^{\mathrm{e}}$ siècle), Traité des noms divins, II, 4, éd. en trad. (du grec) de M. de Gandillac, Paris, 1941.

37. Genèse 1, 26: "faciamus hominem ad imaginem et similitudinem nostram...» (Vulgate).

38. Du moins à partir de la formulation d'Hugues de Saint-Victor à propos des arts mécaniques dans le Didascalicon composé avant 1125 (éd. Ch. H. Buttimer, Washington, 1939, trad. M. Lemoine, Paris: Cerf, 2001) 


\section{RÉFÉRENCES}

N.B. : Les sources, citées en notes, ne sont pas reprises ici.

Abraham-Thisse, S. 2006 La Fraude dans l'industrie textile au Moyen Âge. In G. Béaur, H. Bonin \& Cl. Lemercier (dir.), Fraude, contrefaçon et contrebande, de l'Antiquité à nos jours. Genève: Droz, 431-456.

Andrieu-Guitrancourt, P. 1938 L’Archevêque Eudes Rigaud et la vie de l'Église au XIII ${ }^{e}$ siècle d’après le "Regestrum visitationum ». Paris: Librairie du recueil Sirey.

Bautier, R.-H. 1966 La Place de la draperie brabançonne et plus particulièrement bruxelloise dans l'industrie textile du Moyen Âge, Annales de la Société royale d'archéologie de Bruxelles 51 : 31-63.

Blaise, A. 1975 Lexicon latinitatis medii aevi. Dictionnaire latin-français du Moyen Âge. Turnhout: Brepols.

Calvin, J. 1599 Traité des reliques. Genève: Pierre de la Rouiere.

Cardon, D. 1999 La Draperie au Moyen Âge. Essor d’une grande industrie européenne. Paris: CNRS éditions.

Duby, G. 1996 [1967] L’An Mil, repris dans Féodalité. Paris: Gallimard (Quarto).

Du Cange, Ch. 1883-1886 Glossarium medice et infima latinitatis. Niort: L. Favre.

Espinas, G. 1923 La Draperie dans la Flandre française au Moyen Âge, 2 vol. Paris: Picard.

Espinas, G. \& Pirenne, H. 1906-1966 Recueil de documents relatifs à l'histoire de l'industrie drapière en Flandre, 7 vol. Bruxelles: Palais des Académies.

Favresse, F. 1961 [1955] Les Draperies bruxelloises en 1282. In Études sur les métiers bruxellois au moyen âge. Bruxelles: Université libre de Bruxelles, Institut de sociologie : 95-117.

Feller, L. \& Rodriguez Lopez, A. 2010 Les Objets sous contrainte. Gages, saisies, confiscations, vols, pillages, recel au Moyen Âge, Bulletin du Centre d'études médiévales d'Auxerre 14.

— [En ligne] http://cem.revues.org/index11524.html (consulté le 20/05/2011).

Godefroy, F. 1881-1902 Dictionnaire de l'ancienne langue française et de tous les dialectes du IXe au XVe siècle, 10 vol. Paris: Librairie des Sciences et des Arts.

Gaudemet, J. 1987 Le Mariage en Occident. Les mœurs et le droit. Paris: Cerf.

Ginzburg, C. 2001 [éd. italienne 1998] Représentation. Le mot, l'idée, la chose. In À distance. Neuf essais sur le point de vue en histoire. Paris: Gallimard : 73-88.

Grenier, J.-Y. 1996 L'Économie d'Ancien Régime. Un monde d'échange et d’incertitude. Paris: Albin Michel.

Kusman, D. 2005 Mariage et réseaux financiers internationaux à la fin du xill siècle. La pesanteur des alliances féodales face à la déloyauté des marchés, Francia 32 (1) : 121-156.

Legner, A., 1995 Reliquien in Kunst und Kult. Zwischen Antike und Aufklärung. Darmstadt: Wissenschaftliche Buchgesellschaft.

Le Goff, J. 1999 [1976] Le Rituel symbolique de la vassalité, repris dans Un autre Moyen Âge. Paris: Gallimard, 333-399.

Le Goff, J., Palazzo, É., Bonne, J.-Cl. \& Colette M.-N. 2001 Le Sacre royal à l'époque de saint Louis. D'après le manuscrit latin 1246 de la BNF. Paris: Gallimard.

Lemonnier, P. 2012 Mundane artefacts. Materiality and non-verbal communication. Walnut Creek: Left Coast Press.

Lenain, T. 2008 Du Culte des reliques au monde de l'art. Remarques sur le genèse de la critique d'authenticité, Marburger Jahrbuch für Kunstwissenschaft, 35 : 67-85.

Lenain, T. 2011 Art forgery. The History of a modern obsession. Londres: Reaktion Books.

Méhu, D. (dir.) 2007 Mises en scène et mémoires de la consécration de l'église dans l'Occident médiéval. Turnhout: Brepols.

Obarrio Moreno, J. A. 2009. El proceso por contumacia. Origen, pervivencia y recepción. Madrid: Dykinson.

Palazzo, E. L'Évêque et son image. L'illustration du pontifical au Moyen Âge. Turnhout: Brepols. 
Platelle, H. 1999 Guibert de Nogent et le De pignoribus sanctorum. Richesses et limites d'une critique médiévale des reliques. In E. Bozoky \& A.-M. Helvetius (dir.), Les Reliques. Objets, cultes, symboles. Turhnout: Brepols, 109-122.

Queller, D. E. 1967 The Office of the ambassador in the Middle Ages. Princeton: Princeton University Press. Rey, A. (dir.) 1992 Dictionnaire historique de la langue française. Paris: Dictionnaires le Robert.

Reynolds, R. E. 1983 The Liturgy of clerical ordination in early medieval art, Gesta 22 (1) : 27-38.

Sansterre, J.-M. 2011 Unicité du prototype et individualité de l'image: la Vierge Marie et ses effigies miraculeuses, approche diachronique d'une croyance entre évidence, rejet et ambiguité. In Th. Lenain, J-M Sansterre \& R. Dekoninck (dir), Image et Prototype. Degrés 145-146 : 1-17.

Tobler, A., Lommatzsch, E. \& Helmut, Ch. 1925-2002. Altfranzösisches Wörterbuch, 11 vol. Berlin, Wiesbaden, Stuttgart: Franz Steiner.

Weiss, S. 1995 Die Urkunden der päpstlichen Legaten von Leo IX. bis Coelestin III. 1049-1198. Cologne, Weimar, Vienne: Böhlau.

Wirth, J. 2004 Image et relique dans le christianisme occidental. In P. Borgeaud \& Y. Volokhine (dir.), Les Objets de la mémoire. Bern, Berlin, Bruxelles: P. Lang : 325-342. 


\section{RÉSUMÉ}

Les biens non substituables dans l'Occident médiéval. Certains biens sont indispensables à la vie ou à la reproduction de la société. Le monde médiéval avait des mots particuliers pour exprimer cela, et le caractère irremplaçable d'une chose s'y posa symboliquement dans deux domaines en particulier. Celui tout d'abord du sacré. Certains objets semblent nécessaires au bon déroulement des rituels (mais quels objets et comment le savoir?), tandis que d'autres sont rendus « spéciaux », inaliénables ou différents de leurs semblables par une opération rituelle. Les reliques, elles, étaient d'emblée uniques, mais cette originalité était inlassablement trahie par d'innombrables exemplaires. Dans l'univers économique cette fois, à la fin du Moyen Âge, apparaissent des biens non substituables d'un genre nouveau. Certains objets sont désormais des produits. Ils se caractérisent, précocement dans l'industrie textile, par une «personnalité » forte, une réputation, et des marques qui garantissent leur authenticité. Si bien qu'ils ne peuvent plus être remplacés par d'autres qui s'acquittent pourtant des mêmes fonctions. Nouveauté médiévale, nous connaissons par cœur cet ordre des choses qui nous fait choisir un modèle plutôt qu'une occurrence, et préférer l'original à la copie.

\section{ABSTRACT}

Non substitutable goods in the Medieval West. Goods are deeply essential to life and to the reproduction of society. In Medieval times, people had a specific vocabulary for this fact. The irreplaceability of certain objects was acknowledged at a symbolic level in two domains. One of these was the domain of the sacred : certain objects seem to have been indispensable for the performance of rituals (but what objects were they, and how can we know?), while others were made "special", or different from their fellow objects, through ritual processes. The original uniqueness of relics, on the other hand, was prejudiced by the countless examples that existed of them. The other domain for the symbolic acknowledgement of objects' uniqueness was the economic domain. At the end of the Middle Ages, a new kind of non-substitutable goods appeared : certain objects became products. Quite early on, in the textile industry, they became endowed with strong "personalities" and reputations, and were branded as a guarantee of their authenticity. These objects could not be replaced by other goods with the same function. This medieval novelty became the order of things that we know today, making us attend to the model over the singular occurrence, the original over the copy.

\section{MOTS-CLÉS}

Moyen Âge, artefact, sacré, rituel, christianisme, Europe pré-industrielle, branding, textile, copie

\section{KEYWORDS}

Middle Ages, artefact, sacred, ritual, christianism, pre-industrial Europe, branding, textile, copy 